\title{
Pulmonary Neuroendocrine Tumor Presenting as a Left Pleural Effusion
}

\author{
Miguel A. Ortiz, MD; Gabriela Montes, MD; Onix Cantres, MD; and William Rodríguez, MD, MACP
}

\begin{abstract}
The presence of a symptomatic and recurrent unilateral pleural effusion should alert physicians to consider thoracentesis with mindful use of biomarkers not only for therapeutic purposes, but also for diagnosis of both benign and malignant etiologies.
\end{abstract}

\author{
Miguel Ortiz is a \\ Pulmonary and Critical \\ Care Physician, Gabriela \\ Montes is a Physician, \\ Onix Cantres is a \\ Pulmonologist, and \\ William Rodríguez is \\ Chief, Pulmonary and \\ Critical Care Medicine \\ and Training Program \\ Director, all at VA Caribbean \\ Health Care System in \\ San Juan, Puerto Rico. \\ Correspondence: \\ William Rodríguez \\ (william-rodriguez@va.gov)
}

$\mathrm{N}$ euroendocrine tumors (NETs) account for about $0.5 \%$ of all newly diagnosed malignancies. ${ }^{1}$ Pulmonary NETs are rare, accounting for 1 to $2 \%$ of all invasive lung malignancies and involve about 20 to $25 \%$ of primary lung malignancies. 2,3 Their prevalence has increased by an estimated $6 \%$ per year over the past 30 years. $^{2}$ Nonetheless, the time of diagnosis is frequently delayed because of nonspecific symptoms that may imitate other pulmonary conditions.

In the normal pleural space, there is a steady state in which there is a roughly equal rate of fluid formation and absorption. Any disequilibrium may produce a pleural effusion. Pleural fluids can be transudates or exudates. Transudates result from imbalances in hydrostatic and oncotic pressures in the pleural space. Exudates result primarily from pleural and/or lung inflammation or from impaired lymphatic drainage of the pleural space. Clinical manifestations include cough, wheezing, recurrent pneumonia, hemoptysis and pleural effusions. We present a case of a man who developed a large left pleural effusion with a pathology report suggesting a pulmonary NET as the etiology. Being aware of this rare entity may help improve prognosis by making an earlier diagnosis and starting treatment sooner.

\section{CASE PRESENTATION}

A 90-year-old man with a medical history of arterial hypertension, hyperlipidemia, type 2 diabetes mellitus, coronary artery disease, and vascular dementia presented to the emergency department with hypoactivity, poor appetite, productive cough, and shortness of breath. The patient was a for- mer smoker (unknown pack-years) who quit smoking cigarettes 7 years prior. Vital signs showed sinus tachycardia and peripheral oxygen saturation of $90 \%$ at room air. The initial physical examination was remarkable for decreased breath sounds and crackles at the left lung base. Laboratory findings showed leukocytosis with neutrophilia and chronic normocytic anemia. Chest computed tomography (CT) showed a large left-sided pleural effusion occupying most of the left hemithorax with adjacent atelectatic lung, enlarged pretracheal, subcarinal, and left perihilar lymph nodes (Figure 1).

The patient was admitted to the internal medicine ward with the diagnosis of left pneumonic process and started on IV levofloxacin. However, despite 7 days of antibiotic therapy, the patient's respiratory symptoms worsened. This clinical deterioration prompted pulmonary service consultation. Chest radiography demonstrated an enlarging left pleural effusion (Figure 2). A thoracentesis drained 1.2 L of serosanguineous pleural fluid. Pleural fluid analysis showed a cell count of $947 / \mathrm{cm}^{3}$ with $79 \%$ of lymphocytes, total protein $3.8 \mathrm{~g} / \mathrm{dL}$, lactic dehydrogenase (LDH) level $607 \mathrm{U} / \mathrm{L}$, and glucose level $109 \mathrm{mg} / \mathrm{dL}$. Serum total protein was $6.62 \mathrm{~g} / \mathrm{dL}, \mathrm{LDH} 666 \mathrm{U} / \mathrm{L}$ and glucose $92 \mathrm{mg} / \mathrm{dL}$ (Tables 1 and 2). Alanine transaminase (ALT) and aspartate aminotransferase (AST) were $11 \mathrm{U} / \mathrm{L}$ and 21 U/L, respectively. Using Light criteria, the pleural:serum protein ratio was 0.57 , the pleural:serum LDH ratio was 0.91 , and the pleural LDH was more than two-thirds of the serum LDH. These calculations were 


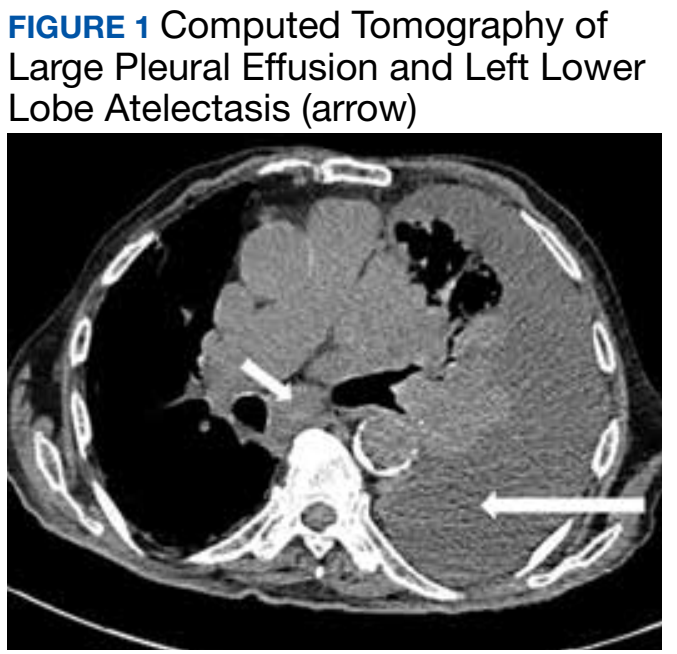

Image shows large left pleural effusion (long arrow) with left hilar and mediastinal lymphadenopathy (short arrow).

consistent with an exudative effusion. An infectious disease workup, including blood and pleural fluid cultures, was negative.

The pleural fluid concentrated cell block hematoxylin and eosin (H\&E) staining showed chromatin, prominent nucleoli, and nuclear molding, which was compatible with high-grade lung NET (Figure 3). The cell block immunohistochemistry (IHC) was positive for synaptophysin, chromogranin A, and neuron specific enolase (NSE) also consistent with a high-grade pulmonary NET (Figure 4). The proliferation marker protein Ki-67 labeling index (LI) showed a proliferation index $>20 \%$ (Figure 5). The patient did not have decision-making capacity given vascular dementia. Multiple attempts to contact the next of kin or family members were unsuccessful. Risks vs benefits were evaluated, and given the patient's advanced age and multiple comorbidities, a conservative management approach under palliative care was chosen. For this reason, further genomic studies were not done.

\section{DISCUSSION}

NETs are a group of neoplasms that differ in site, amount of cell propagation, and clinical manifestations. ${ }^{4}$ These tumors are rare with an estimated incidence of 25 to 50 per $100,000 .{ }^{4}$ The most commonly affected organ systems are the gastroenteropancreatic and the bronchopulmonary tracts, accounting for $60 \%$ and $25 \%$ of the tumors, respectively. ${ }^{4}$ The incidence is in-
FIGURE 2 X-Ray Demonstrating Pleural Effusion (arrow)

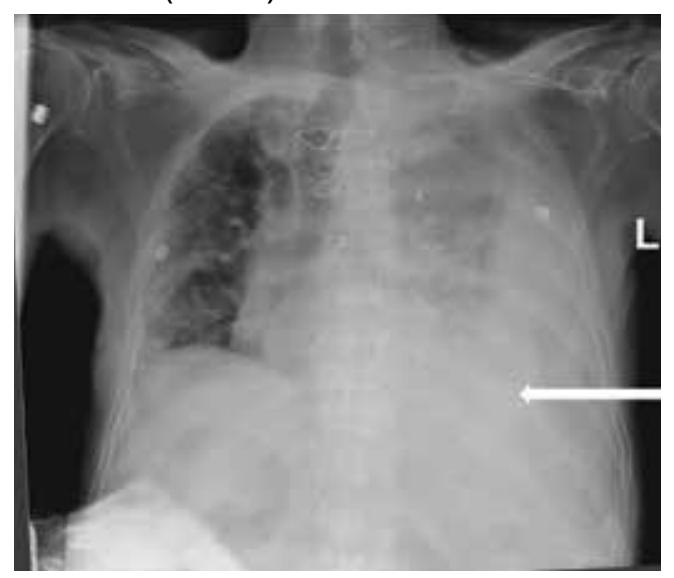

TABLE 1 Pleural Fluid Analysis Cell Count/Differential

\begin{tabular}{lcc}
$\begin{array}{l}\text { Pleural Fluid } \\
\text { Analyses }\end{array}$ & $\begin{array}{c}\text { Result/ } \\
\text { Status }\end{array}$ & $\begin{array}{c}\text { Reference } \\
\text { Range }\end{array}$ \\
\hline Fluid color & Red & Pale yellow \\
\hline Fluid appearance & Turbid & Clear \\
\hline $\begin{array}{l}\text { Supernatant color } \\
\text { appearance }\end{array}$ & Dark yellow & N/A \\
\hline $\begin{array}{l}\text { Supernatant } \\
\text { Cell count, cm }\end{array}$ & Clear & N/A \\
\hline $\begin{array}{l}\text { Polymorphonuclear } \\
\text { cells, \% }\end{array}$ & 947 & $0-1,000$ \\
\hline $\begin{array}{l}\text { Lymphocytes, \% } \\
\text { Macrophages, \% }\end{array}$ & 79 & $<25$ \\
\hline
\end{tabular}

creasing over the past years in part because of novel diagnostic techniques.

The average age of diagnosis is between the fourth and sixth decades, affecting more women than men. ${ }^{5}$ Smoking has been identified as a possible culprit for the development of these neoplasms; nonetheless, the association is still not clear. ${ }^{4}$ For example, poorly differentiated pulmonary NETs have a strong association with smoking but not well-differentiated pulmonary NETs. ${ }^{2}$

Patients typically present with cough, wheezing, hemoptysis, and recurrent pneumonias, which are in part a consequence of obstruction caused by the mass. ${ }^{2}$ Sometimes, obstruction may yield persistent pleural 


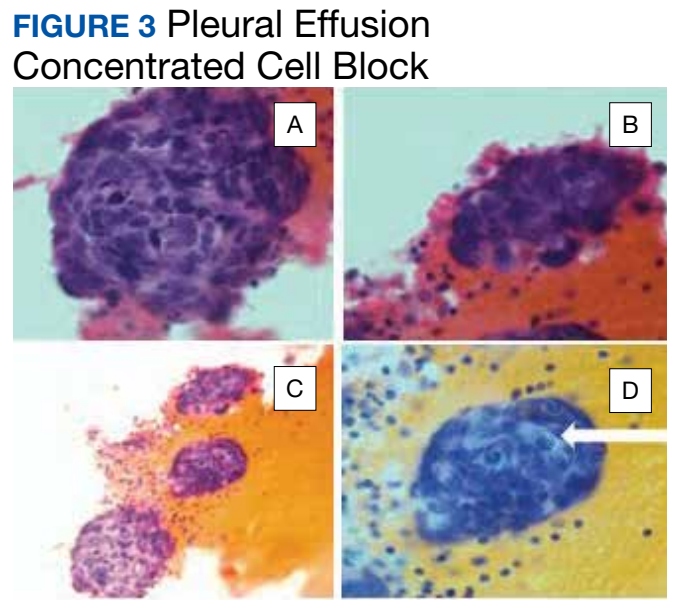

Pleural effusion concentrated cell block showing clusters of malignant cells with clumped "salt \& pepper" (arrow) chromatin, prominent nucleoli and nuclear molding compatible with high-grade neuroendocrine tumor (hematoxylin and eosin stain, magnification x40).

effusions. Hemoptysis may be seen secondary to the vascularity of pulmonary NETs.

The diagnosis is often delayed because patients are frequently treated for infection before being diagnosed with the malignancy, such as in our case. Radiologic image findings include round opacities, central masses, and atelectasis. Pulmonary NETs are frequently found incidentally as solitary lung nodules. The CT scan is the most common diagnostic modality and can provide information about the borders of the tumor, the location and surrounding structures, including the presence of atelectasis. ${ }^{5}$ Pulmonary NETs are usually centrally located in an accessible region for lung biopsy. In cases where the mass is not easily reachable, thoracentesis may provide the only available specimen.

The 2015 World Health Organization classification has identified 4 histologic types of pulmonary NETs, namely, typical carcinoid (TC), atypical carcinoid (AC), large cell neuroendocrine carcinoma (LCNEC) and small cell lung carcinoma (SCLC). ${ }^{6}$ The low-grade pulmonary NET, the typical carcinoid, is slow growing and has lower rates of metastasis. The intermediate-grade NET, the atypical carcinoid, is more aggressive. The highgrade NETs, the LCNEC and the SCLC, are aggressive and spread quickly to other places. ${ }^{6}$ Consequently, LCNEC and SCLC
FIGURE 4 Pleural Effusion Concentrated Cell Block

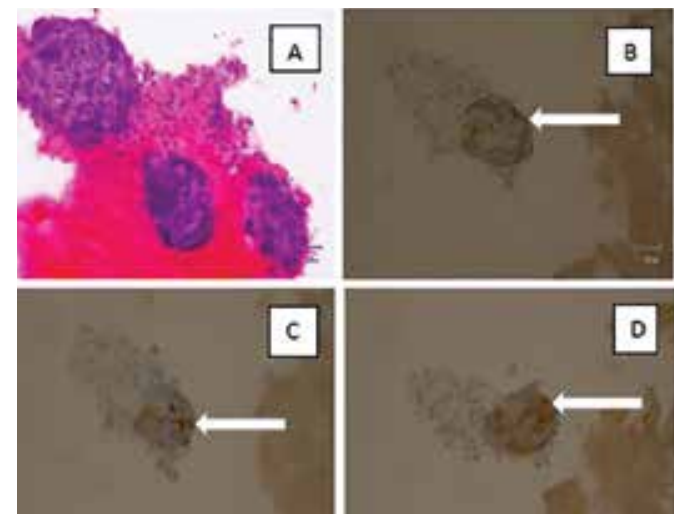

Immunohistochemistry showing positive reaction for neuroendocrine tumor markers (brown staining arrowed). A, Concentrated cell block (hematoxylin and eosin stain, magnification x40); B, Synaptophysin staining; C, Chromogranin A staining; and D, Neuron specific enolase staining.

have higher mortalities with a 5-year survival, ranging from 13 to $57 \%$ and $5 \%$, respectively. ${ }^{7}$

Tumors may be histomorphologically classified by $H \& E$ staining. The main characteristics that differentiate the low- and high-grade NETs are the presence of necrosis and the mitotic rate. Both categories form neuropeptides and have dense granular cores when seen with an electron microscopy. ${ }^{6}$ The TC and AC have welldefined, organized histologic patterns, no necrosis, and scarce mitosis. On the other hand, the LCNEC and SCLC are poorly differentiated tumors with necrosis, atypia, and mitosis. ${ }^{6}$ LCNEC can be separated from SCLC and other tumors by IHC staining, whereas SCLC is primarily distinguished by morphology.

If the biopsy sample size is small, then IHC morphology and markers are helpful for subclassification. ${ }^{8}$ IHC is used to discern between neuroendocrine (NE) vs non-NE. The evaluation of pleural fluid includes preparation of cell blocks. Cell block staining is deemed better for IHC because it mimics a small biopsy that enables superior stains. ${ }^{9}$ The need for a pleural biopsy in cases where the cytology is negative depends on treatment aims, the kind of tumor, and the presence of metastasis. ${ }^{10}$ In almost $80 \%$ of cases, pleural biopsy and cytology are the only specimens obtained for analysis. 
Therefore, identification of these markers is practical for diagnosis. ${ }^{10}$ For this reason, pleural effusion samples are appropriate options to lung biopsy for molecular studies. ${ }^{10}$

Ki-67 LI in samples has the highest specificity and sensitivity for low-tointermediate-grade vs high-grade tumors. It is being used for guiding clinical and treatment decisions. ${ }^{6}$ In SCLC, the Ki-67 LI is not necessary for diagnosis but will be about $80 \% .{ }^{11}$ The tumor cells will show epithelial characteristics with positive cytokeratin AE1/AE3 and monoclonal antibody CAM5.2 and neuroendocrine markers, including NCAM/CD56, chromogranin A, and synaptophysin. ${ }^{11}$

Thyroid transcription factor-1 (TTF1 ) is positive in most cases. In LCNEC, the Ki-67 LI is between $40 \%$ and $80 \%$. NCAM/ CD56, chromogranin A, and synaptophysin are present in 92 to $100 \%, 80$ to $85 \%$, and 50 to $60 \%$, respectively. ${ }^{11}$ TTF-1 is identified in half of the tumors. All these tumors express pancytokeratin (AE1/AE3), cytokeratin 7 or low-molecular-weight cytokeratin. Likewise, the carcinoids will show markers, such as chromogranin A, synaptophysin, CD56, and epithelial markers like pancytokeratin. ${ }^{11}$ However, the high-molecular-weight cytokeratin and TTF-1 are negative. Furthermore, NSE is considered a good tumor marker in the diagnosis and prognosis of SCLC. NSE also has been reported in NSCLC. The level of NSE correlates with tumor burden, number of metastatic sites, and response to treatment. ${ }^{12}$ A potentially useful marker is the insulinoma-associated protein 1 , which is a nuclear determinant of NE differentiation that stains all types of pulmonary NETs irrespective of the histology but does not stain adenocarcinoma or squamous cell carcinoma (SCC). ${ }^{6}$

Recently, genomic studies have identified gene alterations that have become standard of care for diagnosis and targeted therapies. ${ }^{8}$ For example, epidermal growth factor receptor (EGFR) and echinoderm microtubule-associated proteinlike 4, and anaplastic lymphoma kinase (EML4-ALK) mutations have been found in about $25 \%$ of lung adenocarcinomas. ${ }^{8}$ Other abnormalities in LKB1/STK11, NF1, CDKN2A, SMARCA4 and KEAP1, KRAS, MET, ROS1, and RET have also been identified. ${ }^{8}$ On the other
FIGURE 5 Pleural Effusion Concentrated Cell Block Proliferation Marker Ki-67 Labeling Index

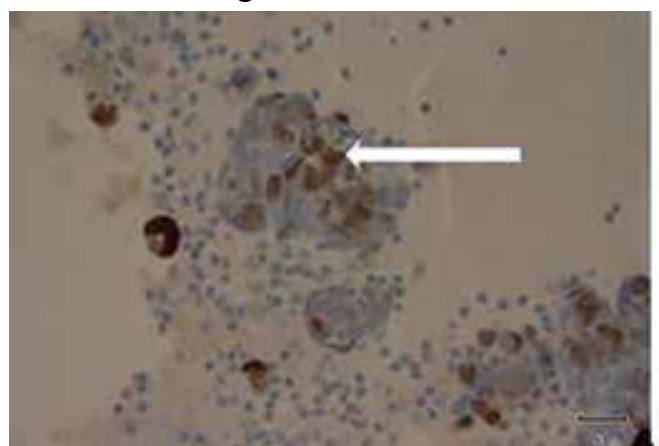

Labeling index showing a proliferation index $>20 \%$ (arrow).

TABLE 2 Pleural Fluid Chemical Analysis

\begin{tabular}{lcc}
$\begin{array}{l}\text { Chemistry Pleural } \\
\text { Fluid/Serum }\end{array}$ & Result & $\begin{array}{c}\text { Reference } \\
\text { Range }\end{array}$ \\
\hline Pleural pH & $\begin{array}{c}\text { Not } \\
\text { available }\end{array}$ & N/A \\
\hline Pleural total protein, g/dL & 3.8 & N/A \\
\hline Pleural LDH, U/L & 607 & N/A \\
\hline Pleural glucose, mg/dL & 109 & N/A \\
\hline Serum LDH, U/L & 666 & $60-200$ \\
\hline Serum total protein, g/dL & 6.62 & $6.0-8.5$
\end{tabular}

Abbreviation: $\mathrm{LDH}$, lactate dehydrogenase.

hand, SCC rarely have derangements in EGFR and EML4-ALK, but do show changes in RTKs, DDR2M, FGGRs, among others. ${ }^{8}$ In TC and AC, observed molecular alterations include MEN1 mutations, mTOR, and SSTRs pathway activation, and GC/ CEACAM1 and CD44/OTP expression. ${ }^{13}$ LCNEC and SCLC have shown TP53 and RB1 mutations and CDX2/VIL1/BAI3 expression. DLL3 expression and MET mutations may be present in SCLC. ${ }^{13}$ Last, chromatin remodeling gene mutations have been identified in all these lung NET types. ${ }^{13}$

Furthermore, neuropeptides and neuroamines may be measured in the blood and urine.${ }^{14}$ Pulmonary NETs may be functional and secrete these substances, leading to systemic symptoms based on the released molecules. ${ }^{15}$ However, pulmonary NETs produce less serotonin than gastrointestinal NETs; therefore, carcinoid syndrome is less 
frequent in pulmonary NETs. ${ }^{16}$ Liver metastasis is often present when it occurs. ${ }^{5}$ Other possible clinical features include Cushing syndrome and acromegaly depending on the secreted hormones. ${ }^{5}$

In a recent metanalysis, serum $\mathrm{LDH}$ has been found to have a prognostic role in Ewing sarcoma, urologic cancers, malignant mesothelioma, among others. ${ }^{17}$ It demonstrated that a higher LDH concentration is associated with worse survival in patients with lung cancer. ${ }^{17}$ Serum LDH is an enzyme that catalyzes the reaction between lactic acid and pyruvic acid that typically takes place in anaerobic conditions. ${ }^{17} \mathrm{LDH}$ levels are elevated in malignancies because tumors have an anaerobic environment. Elevated LDH levels correlate with the anaerobic metabolism in the tumor. Other studies also have noted that patients with high metastatic score have higher LDH levels. ${ }^{17}$ Therefore, LDH may reflect tumor extension.

In addition, other techniques, such as somatostatin-receptor imaging are specifically beneficial in tumors that express the somatostatin receptor. ${ }^{16}$ For this reason, this type of study is typically indicated in patients with known metastasis, not in patients with low-grade tumors. Abdominal CT scans are done because the liver is a common site for metastasis.

Our case report demonstrates how biomarkers help diagnose these potentially aggressive and life-threatening tumors that may present as a common condition such as a pleural effusion. Using a less invasive and quicker approach with thoracentesis rather than with lung biopsies is a diagnostic tool in this entity. IHC in cell blocks is a reasonable diagnostic method especially in patients in whom performing a lung biopsy is difficult.

\section{CONCLUSIONS}

The presence of a symptomatic and recurrent unilateral pleural effusion must urge physicians to consider thoracentesis with mindful use of biomarkers not only for therapeutic purposes, but also for diagnosis of a variety of etiologies, both benign and malignant.

\section{Author disclosures}

The authors report no actual or potential conflicts of interest with regard to this article.

\section{Disclaimer}

The opinions expressed herein are those of the authors and do not necessarily reflect those of Federal Practitioner, Frontline Medical Communications Inc., the US Government, or any of its agencies. This article may discuss unlabeled or investigational use of certain drugs. Please review the complete prescribing information for specific drugs or drug combinations-including indications, contraindications, warnings, and adverse effects-before administering pharmacologic therapy to patients.

\section{References}

1. Oronsky B, Ma PC, Morgensztern D, Carter CA. Nothing but NET: a review of neuroendocrine tumors and carcinomas. Neoplasia. 2017;19(12):991-1002. doi: 10.1016/j.neo.2017.09.002

2. Hendifar AE, Marchevsky AM, Tuli R. Neuroendocrine tumors of the lung: current challenges and advances in the diagnosis and management of well-differentiated disease. J Thorac Oncol. 2017;12(3):425-436. doi: 10.1016/j.jtho.2016.11.2222

3. Fisseler-Eckhoff A, Demes M. Neuroendocrine tumors of the lung. Cancers (Basel). 2012;4(3):777-798. doi: $10.3390 /$ cancers 4030777

4. Mandegaran R, David S, Screaton N. Cardiothoracic manifestations of neuroendocrine tumours. Br J Radiol. 2016;89(1060). doi: 10.1259/bjr.20150787

5. Caplin ME, Baudin E, Ferolla P, et al; ENETS consensus conference participants. Pulmonary neuroendocrine (carcinoid) tumors: European Neuroendocrine Tumor Society expert consensus and recommendations for best practice for typical and atypical pulmonary carcinoids. Ann Oncol. 2015;26(8):1604-1620. doi: 10.1093/annonc/mdv041

6. Pelosi G, Sonzogni A, Harari S, et al. Classification of pulmonary neuroendocrine tumors: new insights. Trans/ Lung Cancer Res. 2017;6(5):513-529. doi: 10.21037/tlcr.2017.09.04

7. Rossi G, Bertero L, Marchiò C, Papotti M. Molecular alterations of neuroendocrine tumours of the lung. Histopathology. 2018;72(1):142-152. doi: 10.1111/his.13394.

8. Osmani L, Askin F, Gabrielson E, Li QK. Current WHO guidelines and the critical role of immunohistochemical markers in the subclassification of non-small cell lung carcinoma (NSCLC): moving from targeted therapy to immunotherapy. Semin Cancer Biol. 2018;52(pt 1):103-109. doi: 10.1016/j.semcancer.2017.11.019

9. Kaur G, Nijhawan R, Gupta N, Singh N, Rajwanshi A. Pleural fluid cytology samples in cases of suspected lung cancer: an experience from a tertiary care centre. Diagn Cytopathol. 2017;45(3):195-201.

10. Porcel JM. Biomarkers in the diagnosis of pleural diseases: a 2018 update. Ther Adv Respir Dis. 2018;12. doi: $10.1177 / 1753466618808660$

11. Kim JY, Hong SM, Ro JY. Recent updates on grading and classification of neuroendocrine tumors. Ann Diagn Pathol. 2017;29:11-16. doi: 10.1016/j.anndiagpath.2017.04.005

12. Isgrò MA, Bottoni $P$, Scatena R. Neuron-specific enolase as a biomarker: biochemical and clinical aspects. Adv Exp Med Biol. 2015;867:125-143. doi: 10.1007/978-94-017-7215-0_9

13. Rossi G, Bertero L, Marchiò C, Papotti M. Molecular alterations of neuroendocrine tumours of the lung. Histopathology. 2018;72(1):142-152. doi: 10.1111/his.13394

14. Eriksson B, Oberg K, Stridsberg M. Tumor markers in neuroendocrine tumors. Digestion. 2000;62(suppl 1):33-38.

15. Melosky B. Low grade neuroendocrine tumors of the lung. Front Oncol. 2017;7:119. doi: 10.3389/fonc.2017.00119

16. Gustafsson Bl, Kidd M, Chan A, Malfertheiner MV, Modlin IM. Bronchopulmonary neuroendocrine tumors. Cancer. 2001;113(1):5-21. https://doi.org/10.1002/cncr.23542

17. Deng T, Zhang J, Meng Y, Zhou Y, Li W. Higher pretreatment lactate dehydrogenase concentration predicts worse overall survival in patients with lung cancer. Medicine (Baltimore). 2018;97(38):e12524 